\title{
BMJ Open Health impacts of seated arm ergometry training in patients with a diabetic foot ulcer: protocol for a randomised controlled trial
}

\author{
Matthew McCarthy (D) , ,2 Thomas Yates, ${ }^{1,2}$ David Webb, ${ }^{3}$ Frances Game, ${ }^{4}$ \\ Laura Gray, ${ }^{1}$ Melanie J Davies ${ }^{1,2}$
}

To cite: McCarthy M, Yates T, Webb D, et al. Health impacts of seated arm ergometry training in patients with a diabetic foot ulcer: protocol for a randomised controlled trial. BMJ Open 2020;10:e039062. doi:10.1136/ bmjopen-2020-039062

- Prepublication history and additional material for this paper are available online. To view these files, please visit the journal online (http://dx.doi. org/10.1136/bmjopen-2020039062).

Received 06 April 2020 Revised 19 May 2020 Accepted 20 May 2020

D Check for updates

(C) Author(s) (or their employer(s)) 2020. Re-use permitted under CC BY-NC. No commercial re-use. See rights and permissions. Published by BMJ.

${ }^{1}$ Diabetes Research Centre, University of Leicester, Leicester, UK

${ }^{2}$ NIHR Leicester Biomedical Research Centre, University of Leicester, Leicester, UK

${ }^{3}$ University Hospitals of Leicester NHS Trust, Leicester, UK

${ }^{4}$ Department of Diabetes and Endocrinology, University Hospitals of Derby and Burton NHS Foundation Trust, Derby, UK

Correspondence to

Dr Matthew McCarthy;

mm636@le.ac.uk

\section{ABSTRACT}

Introduction Once diagnosed with a diabetic foot ulcer (DFU), patients are advised to offload, keeping pressure off the foot in order to facilitate ulcer healing. An increase in offloading is often accompanied by reductions in physical activity which can worsen the overall health of patients. While unable to perform traditional forms of upright activity, one mode of exercise that would allow patients to be physically active while adhering to offloading instruction is seated arm ergometry. The merits of tailored aerobic exercise in DFU remain unexplored.

Methods and analysis This is a prospective openlabel randomised controlled trial. Participants will be randomised to one of two groups, an exercise intervention group or control. The intervention group are required to undertake arm ergometry training at a moderate intensity (65\%-75\% HRpeak), three times per week for 12 weeks as individually prescribed by an exercise physiologist, while the control group will continue to receive standard care alone. Assessment of outcome measures will occur at baseline and after the intervention period, these will include: a seated $\mathrm{VO}_{2}$ peak test, a blood sample, a short physical performance battery, a dual-energy $X$-ray absorptiometry scan and completing a range of health-based questionnaires. The above will be used to determine: cardiorespiratory fitness, metabolic health, physical function, body composition and quality of life, respectively. Ulcer area will also be measured as an approximate marker of ulcer healing.

Ethics and dissemination This trial has been approved by 'Yorkshire \& The Humber-Leeds West Research Ethics Committee' (19/YH/0269). Trial results will be published in peer-reviewed journals and through conference presentations.

Trial registration number ISRCTN16000053. Registered in accordance with WHO Trial Registration Data Set (version 1.3.1).

\section{INTRODUCTION}

Physical activity and exercise are critical for optimal health in those with diabetes ${ }^{1}$ and are the recommended front-line therapy in its management, even after the commencement of hypoglycaemic agents. ${ }^{2}$ Recommendations specific to those with diabetes encourage
Strengths and limitations of this study

- This is the first randomised controlled trial to explore the effects of non-weight bearing aerobic exercise in those with diabetic foot ulceration.

- Our mode of exercise (arm ergometry) can be performed without deviating from current clinical advice to remain offloaded to facilitate ulcer healing.

- Having a standard care control means we can see the benefits to the patient over and above that of their normal treatment alone.

- Other than the addition of an exercise programme (in the intervention group), all participants will be instructed to continue their lives as normal (ie, their dietary and daily activity habits). This is difficult to monitor objectively and will rely on self-report.

adults to engage in $\geq 150$ min of moderateto-vigorous intensity physical activity per week, and to increase total daily incidental (non-exercise) physical activity for additional health benefits. ${ }^{3}$

Due to neuropathic and vascular complications of diabetes, prevalence of diabetic foot ulcer (DFU) is high, reported at between 21 and 33 cases per 1000 persons in an English healthcare setting. ${ }^{4}$ Diabetic foot complications constitute the primary cause of hospitalisation in people with diabetes ${ }^{5}$ and are thought to cost the National Health Service (NHS) between $£ 972$ million and £1.13 billion/year in England alone, ${ }^{6}$ a figure exceeding the combined annual cost of three of the four most common cancers. ${ }^{7}$

Guidelines advise that patients with DFU keep the affected foot offloaded in order to facilitate healing ${ }^{8}$ and clinicians supply offloading footwear as part of standard care to reduced localised stress on the ulcer. ${ }^{9}$ This footwear can create lower limb length discrepancy, ${ }^{10}$ alter normal walking pattern 
and impede postural stability, ${ }^{11}$ increasing risk of falls in those who do not tailor lifestyles accordingly.

Advice to offload, in conjunction with prescribed offloading footwear, leaves patients with DFU unable to engage in 'traditional' upright exercises recommended in global physical activity guidelines such as walking, dancing, gardening and hiking. ${ }^{12}$ Resultantly, many patients may feel that offloading and inactivity are mutually exclusive terms, with restful sitting often deemed the only viable alternative. Another mode of activity suggested in the aforementioned guidelines is swimming; however, this poses other challenges to the open wound such as possible infection and cross contamination. ${ }^{13}$

As per the advice of the American Diabetes Association and the European Association for the Study of Diabetes, it is important to tailor exercise interventions to meet the unique needs of all individuals with diabetes. ${ }^{13}$ Despite this guidance, no studies to our awareness have successfully tailored an aerobic exercise programme to the nonweight bearing requirements of the DFU population. Most research conducted in DFU focuses on treatment of the ulcer itself (ie, topical dressings), and does not account for the broader health status of this population. ${ }^{14}$

One study used a cycling protocol in DFU ${ }^{15}$; however, this mode of exercise still exerts downward pressure on the feet, and depending on the position and severity of an ulcer could aggravate it further, hence continued efforts to create footwear conducive to cycling. ${ }^{16}$ One mode of exercise that tailors to the non-weight bearing requirements of the DFU population is seated arm ergometry. Seated arm ergometry training has led to improvement in the cardiorespiratory fitness level of patients with spinal cord injury who are also under offloading instruction and have low baseline physical activity levels. ${ }^{17}$ Given that cardiovascular disease is the largest contributor to mortality in the DFU population, ${ }^{18}$ improving the efficiency of the cardiovascular system through arm ergometry training holds strong clinical potential. This is especially important given the approximate $50 \%$ mortality rate of this population within 5 years of DFU diagnosis. ${ }^{19-21}$

In addition, arm ergometry exercise has also proven effective in regulating glycaemic control in those at high risk of diabetes. ${ }^{22}$ Poor glycaemic control, anticipated while under offloading instruction, is likely to increase the risk/severity of peripheral artery disease ${ }^{23}$ and peripheral neuropathy ${ }^{24}$ in patients with diabetes, both of which are involved in the pathogenesis of DFUs. ${ }^{25}$

At present, $90 \%$ of individuals with type 2 diabetes are deemed to be overweight or obese. ${ }^{26}$ Further reduction in activity levels on guidance to offload a DFU is likely to reduce daily energy expenditure further, unless alternative ways to remain active are explored.

Another concern among the DFU population is healthrelated quality of life, which is deemed to be significantly worse compared with individuals with diabetes whom do not present with a DFU, ${ }^{27}$ and has been found to predict DFU-related amputation and also mortality. ${ }^{28}$ The impact of regular exercise training on health-related quality of life in people with diabetes has been well documented. ${ }^{2}$

A tailored form of aerobic exercise not only has the potential to improve overall health status of patients with DFU, but may also have positive impacts on the ulcer itself. As core temperature increases with exercise, a temperature threshold is reached at which point skin blood flow begins to rise, ${ }^{29}$ facilitating improvements in oxygen and nutrient delivery to the entire skin surface. ${ }^{30}$ A reduction in oxygen and nutrient delivery to the skin surface is a known risk factor for $\mathrm{DFU},{ }^{31}$ and an ability to improve this, through a tailored non-weight bearing exercise intervention, may assist with ulcer recovery.

We hypothesise that compared to patients with DFU being treated through standard care alone, the addition of an arm ergometry training programme will improve cardiorespiratory fitness, with resulting benefits to glycaemic control, physical function, body composition, quality of life and ulcer healing. This has the potential to reduce the health burden of this vulnerable and highly prevalent population while simultaneously adhering to standard care instruction to simply 'offload' the feet as much as possible.

It is argued that the process of 'offloading' a DFU is 'saving limbs but not lives' ${ }^{14}$; however, with the anticipated benefits of arm ergometry, in conjunction to its offloaded nature, there may be potential to save both.

\section{METHODS AND ANALYSIS \\ Study design}

This is a prospective open-label randomised controlled trial (RCT) with two arms (an arm ergometry exercise intervention group and a standard care control group). All participants will continue to receive standard care for their foot ulcers which does not routinely include arm ergometry. Assessment of outcome measures will be performed at baseline and following a 12-week arm ergometry exercise training intervention, or equivalent time period for the standard care control group (see figure 1, participant flow chart based on the Standard Protocol Items for Randomised Trials (SPIRIT) ${ }^{32}$ and the Consolidated Standards of Reporting Trials (CONSORT) guidelines for transparent reporting of trials ${ }^{33}$ ).

\section{Setting}

This will be a single-centre study located at the Leicester Diabetes Centre, Leicester General Hospital-England, UK, and facilitated by the lifestyle theme of the NIHR Leicester Biomedical Research Centre. All assessments will be conducted by an experienced research team at the Leicester Diabetes Centre which is located directly above the participant's routine foot clinic appointments and has wheelchair access throughout. The exercise intervention itself will be completed both within this facility and also within the patient's home environment.

\section{Participants}

Patients actively being treated for a DFU will be recruited from the Leicester Diabetes Centre's secondary care foot 


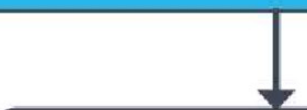

\section{ALLOCATION}

INTERVENTION

\section{FOLLOW-UP}

\section{Baseline assessment visit including:}

- Blood Sample

- Anthropometric measures

- DEXA Scan

- Questionnaires

- Physical Function Tests

- Physical examination

- Blood pressure

- Resting ECG

- Maximal exercise test (incl. ECG)

- Ulcer area measurement (taken at the earliest possible foot clinic appointment)

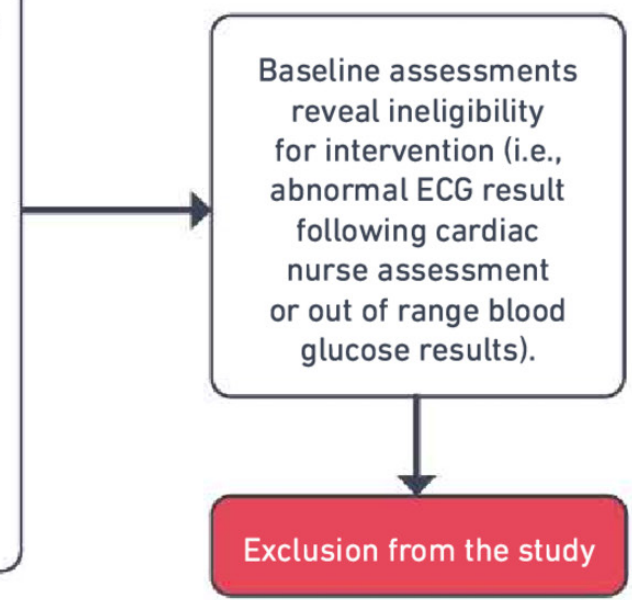
(12 weeks)

7 days habitual activity monitoring

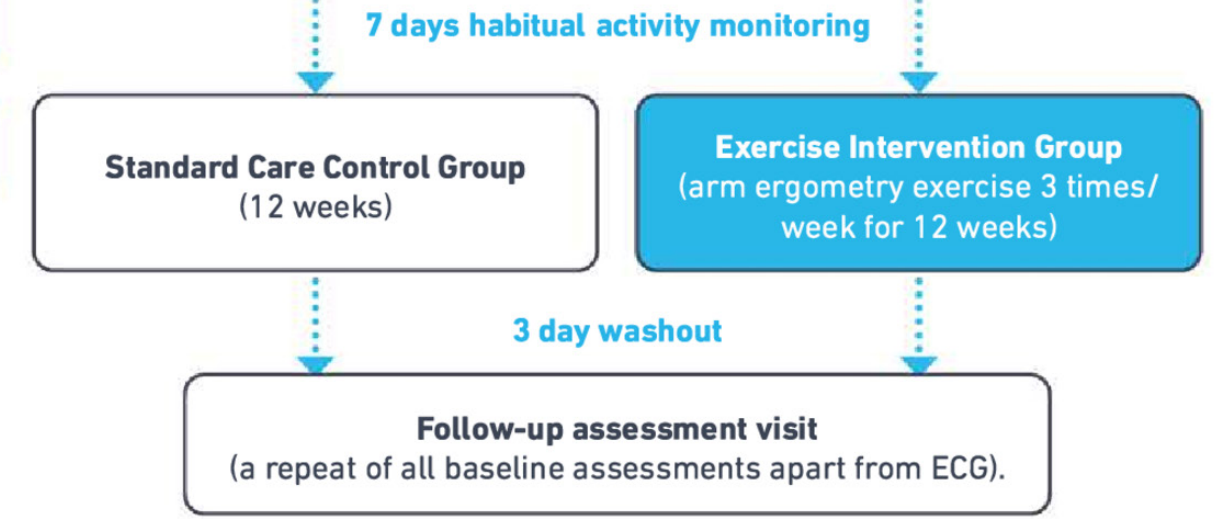

Figure 1 Flow chart depicting flow of participants through the study. DEXA, dual-energy X-ray absorptiometry.

clinic (exact eligibility criteria displayed in table 1). A database containing individuals who have participated in previous research at the Leicester Diabetes Centre and have given consent to be contacted regarding future research will also be used to approach potential participants. All individuals will undergo informed consent (see online supplementary file 1)

\section{Sample size}

Previous research shows that an increase in fitness $\left(\mathrm{VO}_{2}\right.$ peak) of $3.5 \mathrm{~mL} / \mathrm{kg} / \mathrm{min}$ (equivalent to $1 \mathrm{MET}$ ) is associated with a clinically meaningful reduction in all-cause mortality. ${ }^{34}$ An increase of equal or greater than $3.5 \mathrm{~mL} /$ $\mathrm{kg} / \mathrm{min}$ with arm exercise has been shown to be achievable in other clinical populations with low baseline levels of physical activity. ${ }^{17}$
This study was powered $(80 \%)$ to detect a $3.5 \mathrm{~mL} / \mathrm{kg} /$ min difference in the primary outcome of fitness $\left(\mathrm{VO}_{2}\right.$ peak) between baseline and 12 weeks with alpha set at $5 \%$ assuming a SD of $4.0 \mathrm{~mL} / \mathrm{kg} / \mathrm{min} .{ }^{17}$ On this basis, we require 21 participants per group to complete the trial. Therefore, we will recruit 25 individuals to the control group and 30 individuals to the intervention group to allow for a $15 \%$ loss to follow-up and a $15 \%$ non-adherence rate in the exercise intervention. Loss to follow-up and nonadherence rates will be reviewed as the trial progresses, with recruitment numbers revised as required.

\section{Randomisation}

All participants will be randomised to either the standard care control or the exercise intervention. Randomisation (using blocking) will be stratified by sex and whether 


\begin{tabular}{|c|c|}
\hline Inclusion criteria & Exclusion criteria \\
\hline $\begin{array}{l}\text { Actively receiving DFU treatment. } \\
\text { Aged between } 18 \text { and } 75 \text { inclusive. } \\
\text { Able to undertake upper body arm exercise (specifically } \\
\text { arm ergometry). } \\
\text { Deemed safe to exercise further to cardiac nurse evaluation } \\
\text { at baseline (see section } 8.2 .4 \text { ). } \\
\text { Participant is willing to give informed consent to take part } \\
\text { in the study. }\end{array}$ & $\begin{array}{l}\text { Uncontrolled hyperglycaemia (HbA1c }>10 \% \text { - confirmed } \\
\text { through baseline blood sample results or access to recent } \\
\text { (within } 3 \text { months) blood results). } \\
\text { Report taking part in regular (at least once a week) } \\
\text { strenuous sport or activities. } \\
\text { Existing heart problem (a cardiovascular event within last } 12 \\
\text { months or screened by cardiac nurse at baseline). } \\
\text { Underweight or with a body mass index } \leq 18.5 \mathrm{~kg} / \mathrm{m}^{2} \text {. } \\
\text { Comorbidity that the research team consider to be a } \\
\text { contraindication to their study involvement. } \\
\text { Unable to communicate in English sufficiently to provide } \\
\text { consent and participate. }\end{array}$ \\
\hline
\end{tabular}

DFU, diabetic foot ulcer.

ischaemia is present in the foot to ensure even distribution of these characteristics between the study groups, as both could act as confounding variables, skewing the outcome data. Specifically, once baseline assessments have been completed, each participant will be assigned to one of four unique stratification codes that identify them as: (1) ischaemic and male; (2) ischaemic and female; (3) non-ischaemic and male or (4) non-ischaemic and female. These four groups will each have a folder containing sealed envelopes with their allocated group written inside them. Working through the envelopes systematically in each folder will guarantee that for every four individuals within a certain stratification code, two will be allocated to the control and two will be allocated to the intervention (block size of 4).

These folders will be created by the lead study statistician and to maintain allocation concealment, a separate individual will unveil the contents of each envelope to the participant.

\section{Blinding}

It is not possible to blind those in receipt and those delivering the intervention of group allocation. Study participants will be requested not to discuss treatment allocation at their follow-up assessment, where a research assistant blinded to group allocation will be responsible for collecting primary and secondary outcome data. Importantly, blood samples used to determine cardiometabolic health will be analysed by an independent pathology team. There are no circumstances under which it would be necessary to unblind outcome assessors.

\section{Intervention}

Participants allocated to the intervention group will receive a 12-week individualised exercise programme by the treating exercise physiologist. Participants will be encouraged to complete three exercise sessions per week using arm ergometers set-up in Leicester Diabetes Centre's gym facility and in their home environment. Each session will work up to at least $30 \mathrm{~min}$ in duration where deemed appropriate by the physiologist. As encouraged by the American Diabetes Association, ${ }^{3}$ efforts will be made to stagger exercise training sessions so that there are no more than 2 days between them; this maximises potential for glycaemic improvement given the 48 hours of raised insulin sensitivity following exercise. ${ }^{35}$

It is advised by the 'American College of Sports Medicine' that exercise should be performed at $>64 \%$ of peak heart rate in order to induce enough cardiovascular strain to elicit a training response. ${ }^{36}$ As such, exercise physiologists will prescribe participants with target heart rates representative of between $65 \%$ and $75 \%$ of their peak heart rate which will be determined during their baseline assessment visit. Continuous Polar Heart Rate monitoring will allow the exercise physiologist to observe heart rate outputs on a regular basis and adapt the exercise prescription accordingly to ensure heart rates remain within this $65 \%-75 \%$ target. For instance, we anticipate increases in intensity through speed and resistance manipulation will be necessary to reach the $65 \%-75 \%$ target as an individual's fitness improves. Where deemed feasible, duration of exercise prescription will progress up to $150 \mathrm{mins} /$ week ( $50 \mathrm{mins} / \mathrm{session}$ ), in line with physical activity guidelines. ${ }^{12}$

Exercise will be performed through a mixture of supervised sessions at the Leicester Diabetes Centre and unsupervised sessions within the participant's home environment. In order to facilitate home-based exercise, lightweight table-top arm ergometers will be installed in a safe and practical location by members of the study team. For the first 6 weeks, we encourage at least two of the 3 weekly exercise sessions to be performed while under supervision, and for the latter 6 weeks we encourage at least one of the three exercise sessions to be performed under supervision. However, importantly, the exercise physiologist prescribing each exercise session will judge this on an individual basis taking into account participants training progression, supervisional requirements and preferences. 
Participants allocated to the control group will continue their lives as normal and will receive standard care. Both groups will be asked to keep their diet constant throughout.

\section{Exercise intervention adherence}

If a participant fails to partake in more than two-thirds of their prescribed exercise sessions $(n=24)$ they will be deemed to be non-adherent and excluded from the perprotocol analysis. Breaks of up to 2 weeks in total (ie, 6 sessions) will be allowed to accommodate illnesses or holidays with the missed sessions added to the end of the planed intervention period (ie, 12 weeks plus number of missed sessions). During supervised exercise sessions, adherence to exercise prescription will be observed first hand. In terms of the unsupervised sessions (within the home environment), these will rely on self-reported adherence and will be further verified by heart rate data captured on Polar Heart Rate monitors, of which are issued to participants in the exercise intervention group and are required to be worn during all exercise sessions. Any incapacity that arises or non-adherence would lead to early termination of the trial for a given participant.

In order to promote participant adherence and study retention, parking permits and travel reimbursements of up to $£ 10 /$ visit will also be offered to participants for each supervised exercise session to eliminate financial burden.

\section{Study assessments and outcomes}

At baseline, participant characteristics will be collected and will be reported by group, including: sex, ethnicity, medication status, smoking status and employment status.

Assessment of all outcome measures shown below will be taken pre and post the exercise intervention or control period.

The primary outcome of this study is to observe change in cardiorespiratory fitness $\left(\mathrm{VO}_{2}\right.$ peak $)$. This will be determined via an incremental maximal exercise test performed using an arm ergometer. Due to different power capabilities, two different protocols will be used for men and women. ${ }^{38}$ Men will commence at a workload of $30 \mathrm{~W}$ and women at $20 \mathrm{~W}$. In both protocols, the crank rate will be maintained at $70 \mathrm{rev} / \mathrm{min}$ and power requirements increased as a linear ramp at a rate of $10 \mathrm{~W} / \mathrm{min}$ and $6 \mathrm{~W} / \mathrm{min}$ for men and women, respectively (as per exercise testing guidance).$^{38}$ All participants will receive encouragement to continue with this progressive exercise for as long as possible subject to the satisfaction of the cardiac nurse that the patient is fit to continue. Inability to maintain a crank rate above $60 \mathrm{rev} / \mathrm{min}$ will result in termination of the test.

All efforts will be made to stick to this exercise testing protocol, although reductions in speed prescription may be necessary for individuals who cannot achieve the $70 \mathrm{rev} / \mathrm{min}$ arm speed from the offset. The validity of each $\mathrm{VO}_{2}$ peak test will be judged on satisfying $\geq 2$ of the following criteria issued by the British Association of Sports and Exercise Sciences ${ }^{39}$ :
- Exercise test termination at the point of volitional exhaustion.

- Heart rate within $10 \%$ of their age predicted maximum (220 minus age (minus an additional 30 beats/min for beta blockers usage, if necessary)) .

- Respiratory exchange ratio of $\geq 1.15$ towards the latter stages of the test.

Throughout the test, gas will be sampled continuously and analysed using a Metalyser 3B gas analyser (Cortex 3B, Cortex Biophysik, Leipzig, Germany). Peak oxygen consumption $\left(\mathrm{VO}_{2}\right.$ peak) will be calculated using the highest 10 breath average throughout the testing period.

Secondary outcomes will reflect changes in the following:

\section{Cardiometabolic health}

Assessed by HbA1c, glucose, insulin, blood lipids (total cholesterol, high-density lipoprotein cholesterol, nonesterified fatty acids and triglycerides) and creatinine. These will be taken from venous blood while under fasting conditions and will be analysed by the University Hospitals of Leicester pathology department. Blood pressure (BP) will be assessed using an automated BP monitor (Omron Healthcare Europe). Participants will be asked to sit quietly and relax prior to having their BP measurements taken and three readings will be taken, with the average of the last two readings used in the analyses.

\section{Quality of life}

Assessed through a number of questionnaires. First, the Cardiff Wound Impact Schedule that has been validated in a DFU population. ${ }^{40}$ The 36 -Item Short Form Health Survey questionnaire will also be used, ${ }^{41}$ as this is the most common health-related quality of life assessment tool in a DFU population. ${ }^{42}$ The EuroQol-5Dimension5Level (EQ5D-5L) instrument will be used as a measure of self-reported health status. ${ }^{43}$ Hospital Anxiety and Depression Scale (HADS) ${ }^{44}$ will also be used, focusing on non-physical symptoms to assess anxiety and depression. Finally, the Diabetes Distress Scale-17 will be used to assess emotional distress linked to diabetes management, healthcare and treatment regimen. ${ }^{45}$

\section{Physical function}

Lower body physical function will be captured by the short physical performance battery (SPPB). SPPB consists of three parts: the balance test, the gait speed test and the chair stand test. ${ }^{46}$ This test is a widely used instrument for assessing lower extremity function and has a good reproducibility and test-retest reliability. ${ }^{47}$ All patients with DFU will be mobilised through offloading footwear prior to undertaking the performance battery as a safety precaution and if a participant is unable or uncomfortable with certain parts of the SPPB, this will be taken note of and will not be undertaken.

Grip strength will be measured using a digital handheld dynamometer. Participants will be asked to grip the device as hard as possible three times on each side, 
with the elbow flexed at a right angle and the forearm in neutral position (as recommended by American Society of Hand Therapists ${ }^{48}$ ). This test will be undertaken while the participant remains seated. The maximum of the readings generated for each hand is taken as the maximum grip strength.

Upper body strength will also be measured through a $30 \mathrm{~s}$ arm curl test. This test involves lifting a dumbbell (5 lbs for women and $8 \mathrm{lbs}$ for men) as many times in $30 \mathrm{~s}$ as possible and forms part of the 'Senior Fitness Test' performance battery. ${ }^{49}$ The participant sits on a chair, holding the dumbbell in their hand with palms facing towards the body and arm fully extended. With the upper arm braced against the body, participants will lift the lower arm gradually turning the palm up during the upward phase (flexion with supination). The arm should then be lowered back to the original position. This test will be conducted in both left hand and right hand. Finally, the Medical Research Council (MRC) breathlessness scale will be used to grade the effect of breathlessness on daily activities. ${ }^{50}$

\section{Body composition}

Assessed with dual-energy X-ray absorptiometry (DEXA) scanning which will derive a breakdown of: bodily fat, muscle mass and bone density. DEXA scans use ionising radiation to measure different body compartments and are the current reference standard for assessing body composition. $^{51}$ Body weight (Tanita TBE 611: Tanita, West Drayton, UK), waist circumference (midpoint between lower costal margin and iliac crest) and height will also be measured to the nearest $0.1 \mathrm{~kg}, 0.5$ and 0.5 $\mathrm{cm}$, respectively. Arm circumference will be measured on both arms (at the midpoint between the shoulder and the elbow joint), to the nearest $0.5 \mathrm{~cm}$ while the arm is hanging loose.

\section{The cross-sectional area of the foot ulcer}

Determined by an acetate grid tracing method ${ }^{52}$ whereby a clinician based in the secondary care foot clinic will trace around the outer edge of the participants wound onto disposable grid paper and take a photograph of this drawing. The photograph will then be uploaded to software called 'Image J', which will then automatically calculate the ulcer area $\left(\mathrm{cm}^{2}\right)$ and demonstrates high intrareliability and inter-reliability when used to measure the area of DFUs. ${ }^{52}$

\section{Physical activity}

A GENEActiv accelerometer will be worn on the nondominant wrist of each participant 24 hours/day for 7 consecutive days following the baseline assessment visit to quantify physical activity levels prior to the intervention. The GENEActiv device has been found to be a valid and reliable objective measure of physical activity and sedentary behaviour in adults. ${ }^{53}$

\section{Safety considerations}

All participants will be assessed by a cardiac nurse for existing heart problems at baseline via a 12 lead ECG assessment. This will be done both at rest and during the maximal exercise testing. It will be used to verify exercise safety. Once deemed safe to exercise by the cardiac nurse, this will allow participants to exercise both while under supervision and while unsupervised within their home environment (providing they stick to the prescribed exercise for each session, which will have been tailored to the individual's ability levels by an exercise physiologist). Prescribed target heart rates during sessions will also be equivalent to 'moderate' intensity, ensuring that risk of overexertion is minimised.

There is a small possibility that episodes of hypoglycaemia may occur during or after exercise. We will advise all participants taking insulin to self-check their blood glucose levels prior to all exercise sessions. Those with a blood glucose level of $<5 \mathrm{mmol} / \mathrm{L}$ will be advised to consume carbohydrate and told to reassess their blood glucose again before exercise. Participants taking insulin will also be given tools to self-monitor their blood glucose levels following exercise, and will be asked to complete a hypoglycaemia diary. Occurrences of hypoglycaemia will be investigated by the study medic with reduction to insulin therapy prescribed if required.

Although seated arm cycling is deemed as non-weight bearing, it is inevitable that a small amount of downward pressure will be put on the feet in order to maintain body stability throughout the exercise, as such we will advise each participant to rest their feet/footwear on a soft cushion during the exercise to further relieve ground contact pressure.

\section{Adverse event reporting}

All adverse events (AEs) occurring during the study (ie, pain or injuries), whether or not attributed to study, will be recorded on in the case report form (CRF). The following information will be recorded: description, date of onset and end date, severity, assessment of relatedness to study, other suspect device and action taken. AEs considered related to the study as judged by a medically qualified investigator or the sponsor will be followed until resolution or the event is considered stable. All related serious AEs must be reported to the sponsor within one working day of discovery or notification of the event. The CI shall submit an annual report to the ethics committee which lists all AEs that have occurred during the preceding 12 months. Decision of participant or study discontinuation will be taken by the principal investigator.

\section{Statistical analysis}

The aim of this investigation is to compare the change in primary and secondary outcomes pre and post between the exercise intervention group and the standard care control group. The primary analysis will be a 'per-protocol' analysis as this is a proof of principle study where we are primarily interested in the size of the treatment effect, rather than the practicability of the intervention. Per-protocol analysis will be applicable to all participants in the intervention group completing/ 
attending more than two-thirds of their scheduled exercise sessions (ie, $\geq 24$ sessions). All control participants will be included. The analysis will be undertaken on those with complete follow-up data. A CONSORT diagram will be produced. At baseline and follow-up, descriptive variables and outcome measures will be summarised for each group using median (IQR) for continuous variables and count (percentage) for categorical variables. The primary and secondary outcomes will be analysed as difference between groups in change from baseline to follow-up adjusting for baseline (missing baseline data replaced using the indicator method) and randomisation stratification factors within a generalised linear model. The distribution of change for each outcome will be inspected; a linear distribution with an identity link will be used for continuous data that have a normal distribution. Alternative model specifications will be tested for non-parametric data with the method that has the best model fit selected and taken forward for analysis.

A complete case analysis for the primary outcome will also be reported including all individuals in the intervention group with follow-up data regardless of their degree of adherence (intention to treat). A sensitivity analyses for the primary outcome will also investigate the impact of missing data using multiple imputation or another appropriate approach to impute missing data.

\section{Data management}

Collected data will be kept confidential in participant CRFs and later transferred into electronic form (Microsoft Excel 2013). The chief investigator (MJD) is responsible for maintenance of safely kept records and backup of data. Statistical analyses will be performed using SPSS V.24.0.

\section{Patient and public involvement}

Focus groups held at the Leicester Diabetes Research Centre with patients with DFU in preparation for this trial identified 'solutions to not being able to exercise' as a priority. Positive attitudes towards lifestyle interventions, in conjunction with feelings of hopelessness regarding a solution that allows the patients to safely exercise, were a recurring theme.

From our patient and public involvement strategy stemmed a priority to expand research into adaptive exercise in the DFU population, as reflected in this protocol. Pilot data collected from 34 patients with DFU in preparation for the outlined protocol confirmed the low levels of physical activity in this population, strengthening the case for an exercise focused intervention. Furthermore, two individuals who themselves have experienced diabetic foot complications were actively involved in the development of this protocol.

\section{ETHICS AND DISSEMINATION}

The protocol version for this trial is version 1.0-dated the 24 June 2019. The protocol for the RCT follows the
SPIRIT statement guidelines ${ }^{32}$ and is registered with the International Standard Randomised Controlled Trial Number (REF: ISRCTN16000053). The 'University of Leicester' is the sponsor and will have overall responsibility for governance of the study. The University of Leicester Sponsors will have no direct role in study design, data collection, management, analysis, interpretation of data, report writing or publications that arise from the study. This protocol was accepted by 'Yorkshire \& The Humber-Leeds West Research Ethics Committee' in September 2019.

The research team will assist the chief investigator in delivering the study, and will be responsible for: monitoring milestones and targets, data, safety, recruitment, reviewing and interpreting the results and reporting Adverse Events (AE's) and Adverse Reactions (AR's) aligned with local governance requirements.

Potential participants will be informed of all study procedures prior to participation, both verbally and in writing. Confidentiality, voluntariness and freedom to withdraw from the study at any point will be stated. Written informed consent will be obtained from all participants by a research assistant. Any amendments to approved study documentation will be resubmitted to the appropriate parties for reapproval prior to implementation. If a participant wishes to make a complaint, the standard NHS complaint system will be available to them and the University of Leicester sponsor indemnity arrangements are in place in the unlikely event that a participant is harmed due to study negligence.

This trial is subject to the University of Leicester sponsor's risk-based audit programme. An audit trail will be maintained throughout the lifetime of the study and all study-related documentation will be made available for sponsor monitoring, and/or any external audits (ie, Research Ethics Committee).

To maintain participant confidentiality, study participants will receive a unique study Identification at inclusion. Collected data will be coded and stored in the participant CRF with matching study ID; this is considered source data and will only be handled by authorised people while kept in a locked archive at the Diabetes Research Centre-University of Leicester. Participant identifiable information such as name and address will be held in a separate locked archive for participant contact use only. All research data generated by the study will be stored for 5 years, after which it will be destroyed. Providing consent is obtained, blood samples will be stored in a Leicester Diabetes Centre $-80^{\circ} \mathrm{C}$ freezer for up to 10 years in a Human Tissue Authority (HTA) licensed area for future analysis.

The results of this trial will be published in peerreviewed journals and through educational and conference presentations. Primary results and datasets will be available from the corresponding author on reasonable request and results will be reported on group-level only. 
Acknowledgements The authors would like to thank all of their PPIs who gave a unique insight into living with a diabetic foot ulcer, particularly John Barraclough and David Batchelor who helped in discussions surrounding the study procedures and helped them to develop their lay material in preparation for this study. They would also like to thank members of the foot clinic team at the Leicester Diabetes Centre for agreeing to distribute study information to their patients.

Contributors MM, TY, FG, MJD and DW contributed to the study design. LG provided overview of all statistical input. MM secured funding and obtained ethical approval for the study and also drafted the manuscript. TY, FG, MJD and DW critically revised and approved the final manuscript.

Funding This work is supported by Diabetes UK (grant number 18/0005883) and Novo Nordisk UK Research Foundation (Charity Registration number 1056410).

Competing interests None declared.

Patient and public involvement Patients and/or the public were involved in the design, or conduct, or reporting, or dissemination plans of this research. Refer to the Methods section for further details.

Patient consent for publication Not required.

Provenance and peer review Not commissioned; externally peer reviewed.

Open access This is an open access article distributed in accordance with the Creative Commons Attribution Non Commercial (CC BY-NC 4.0) license, which permits others to distribute, remix, adapt, build upon this work non-commercially, and license their derivative works on different terms, provided the original work is properly cited, appropriate credit is given, any changes made indicated, and the use is non-commercial. See: http://creativecommons.org/licenses/by-nc/4.0/.

ORCID iD

Matthew McCarthy http://orcid.org/0000-0002-6328-5168

\section{REFERENCES}

1 Davies MJ, D'Alessio DA, Fradkin J, et al. Management of hyperglycaemia in type 2 diabetes, 2018. A consensus report by the American diabetes association (ADA) and the European association for the study of diabetes (EASD). Diabetologia 2018;61:2461-98.

2 Colberg SR, Sigal RJ, Fernhall B, et al. Exercise and type 2 diabetes: the American College of sports medicine and the American diabetes association: joint position statement executive summary. Diabetes Care 2010;33:2692-6.

3 Colberg SR, Sigal RJ, Yardley JE, et al. Physical Activity/Exercise and diabetes: a position statement of the American diabetes association. Diabetes Care 2016;39:2065-79.

4 Paisey RB, Abbott A, Paisey CF, et al. Diabetic foot ulcer incidence and survival with improved diabetic foot services: an 18-year study. Diabet Med 2019;36:1424-30.

5 Boulton AJ. The diabetic foot: a global view. Diabetes Metab Res Rev 2000;16 Suppl 1:S2-5.

6 Kerr M. Improving footcare for people with diabetes and saving money: an economic study in England. Scotland: Diabetes UK, 2017 https://diabetes-resources-production.s3-eu-west-1.amazonaws. com/diabetes-storage/migration/pdf/Improving\%2520footcare\% 2520economic\%2520study\%2520\%28January\%25202017\%29.pdf

7 Kerr M. Foot Care in Diabetes: The Human and Financial Cost Insight Health Economics for Diabetes UK, 2017. Available: http:// www.londonscn.nhs.uk/wp-content/uploads/2017/04/dia-foot-caremtg-kerr-27042017.pdf [Accessed 10 Mar 2020]

8 Snyder RJ, Frykberg RG, Rogers LC, et al. The management of diabetic foot ulcers through optimal off-loading: building consensus guidelines and practical recommendations to improve outcomes. $J$ Am Podiatr Med Assoc 2014;104:555-67.

9 Cavanagh PR, Lipsky BA, Bradbury AW, et al. Treatment for diabetic foot ulcers. Lancet 2005;366:1725-35.

10 Nahas MRE-, Gawish HM, Tarshoby MM, et al. Effect of simulated leg length discrepancy on plantar pressure distribution in diabetic patients with neuropathic foot ulceration. $J$ Wound Care 2011:20:473-7.

11 van Deursen R. Footwear for the neuropathic patient: offloading and stability. Diabetes Metab Res Rev 2008;24 Suppl 1:S96-100.

12 World Health Organization. World Health organization global recommendations on physical activity for health. Geneva, Switzerland: WHO, 2010. http://www.who.int/dietphysicalactivity/ factsheet_recommendations/en/

13 Barna Z, Kádár M. The risk of contracting infectious diseases in public swimming pools. A review. Ann Ist Super Sanita 2012;48:374-86.
14 Dhatariya K, Fox M. Should patients with active foot ulcers be nonweight bearing or take exercise to improve cardiovascular fitness? Diabetic Foot Journal 2014;17:92-9.

15 Nwankwo MJ, Okoye GC, Victor EA, et al. Effect of twelve weeks supervised aerobic exercise on ulcer healing and changes in selected biochemical profiles of diabetic foot ulcer subjects. Int J Diabetes Res 2014;3:41-8.

16 Crews RT, Smith SR, Ghazizadeh R, et al. Preliminary evaluation of a cycling Cleat designed for diabetic foot ulcers. J Am Podiatr Med Assoc 2017;107:475-82.

17 Valent L, Dallmeijer A, Houdijk H, et al. The effects of upper body exercise on the physical capacity of people with a spinal cord injury: a systematic review. Clin Rehabil 2007;21:315-30.

18 Morbach S, Furchert H, Gröblinghoff U, et al. Long-term prognosis of diabetic foot patients and their limbs: amputation and death over the course of a decade. Diabetes Care 2012;35:2021-7.

19 National Institute for Health and Clinical Excellence. Diabetic foot problems: prevention and management; NICE guideline NG19. 2015. Available: https://www.nice.org.uk/guidance/ng19/chapter/ Introduction [Accessed 10 Mar 2020].

20 Moulik PK, Mtonga R, Gill GV. Amputation and mortality in newonset diabetic foot ulcers stratified by etiology. Diabetes Care 2003;26:491-4.

21 Armstrong DG, Wrobel J, Robbins JM. Guest editorial: are diabetesrelated wounds and amputations worse than cancer? Int Wound J 2007;4:286-7.

22 McCarthy M, Edwardson CL, Davies MJ, et al. Breaking up sedentary time with seated upper body activity can regulate metabolic health in obese high-risk adults: a randomized crossover trial. Diabetes Obes Metab 2017;19:1732-9.

23 Heikkinen M, Salmenperä M, Lepäntalo A, et al. Diabetes care for patients with peripheral arterial disease. Eur J Vasc Endovasc Surg 2007;33:583-91.

24 Pop-Busui R, Boulton AJM, Feldman EL, et al. Diabetic neuropathy: a position statement by the American diabetes association. Diabetes Care 2017;40:136-54.

25 Mishra SC, Chhatbar KC, Kashikar A, et al. Diabetic foot. BMJ 2017;359:j5064.

26 Public Health England. Adult obesity and type 2 diabetes, 2014 Available: https://www.gov.uk/government/publications/adultobesity-and-type-2-diabetes [Accessed 10 Mar 2020].

27 Ribu L, Hanestad BR, Moum T, et al. A comparison of the healthrelated quality of life in patients with diabetic foot ulcers, with a diabetes group and a nondiabetes group from the general population. Qual Life Res 2007;16:179-89.

28 Siersma V, Thorsen H, Holstein PE, et al. Health-related quality of life predicts major amputation and death, but not healing, in people with diabetes presenting with foot ulcers: the Eurodiale study. Diabetes Care 2014;37:694-700.

29 Simmons GH, Wong BJ, Holowatz LA, et al. Changes in the control of skin blood flow with exercise training: where do cutaneous vascular adaptations fit in? Exp Physiol 2011;96:822-8.

30 Bentov I, Reed MJ. Anesthesia, microcirculation, and wound repair in aging. Anesthesiology 2014;120:760-72.

31 Boyko EJ, Ahroni JH, Stensel V, et al. A prospective study of risk factors for diabetic foot ulcer. The Seattle diabetic foot study. Diabetes Care 1999;22:1036-42.

32 Chan A-W, Tetzlaff JM, Altman DG, et al. Spirit 2013: new guidance for content of clinical trial protocols. Lancet 2013;381:91-2.

33 Moher D, Hopewell S, Schulz KF, et al. Consort 2010 explanation and elaboration: updated guidelines for reporting parallel group randomised trials. Int J Surg 2012;10:28-55.

34 Ross R, Blair SN, Arena R, et al. Importance of assessing cardiorespiratory fitness in clinical practice: a case for fitness as a clinical vital sign: a scientific statement from the American heart association. Circulation 2016;134:e653-99.

35 Holtz KA, Stephens BR, Sharoff CG, et al. The effect of carbohydrate availability following exercise on whole-body insulin action. Appl Physiol Nutr Metab 2008;33:946-56.

36 Garber CE, Blissmer B, Deschenes MR, et al. American College of sports medicine position stand. quantity and quality of exercise for developing and maintaining cardiorespiratory, musculoskeletal, and neuromotor fitness in apparently healthy adults: guidance for prescribing exercise. Med Sci Sports Exerc 2011;43:1334-59.

37 Haskell WL, Lee I-M, Pate RR, et al. Physical activity and public health: updated recommendation for adults from the American College of sports medicine and the American heart association. Circulation 2007;116:1081.

38 Mitropoulos A, Gumber A, Crank H, et al. Validation of an arm crank ergometer test for use in sedentary adults. J Sports Sci Med 2017;16:558. 
39 Eston R, Reilly T. Kinanthropometry and exercise physiology laboratory manual. . 3rd ed, 2009: Vol.2. p.178.

40 Price P, Harding K. Cardiff wound impact schedule: the development of a condition-specific questionnaire to assess health-related quality of life in patients with chronic wounds of the lower limb. Int Wound J 2004;1:10-17.

41 Saris-Baglama RN, Dewey CJ, Chisholm GB, et al. QualityMetric health outcomes ${ }^{\mathrm{TM}}$ scoring software 4.0. 138. Lincoln, Rl: QualityMetric Incorporated, 2010.

42 Wukich DK, Raspovic KM. Assessing health-related quality of life in patients with diabetic foot disease: why is it important and how can we improve? the 2017 Roger E. Pecoraro Award Lecture. Diabetes Care 2018;41:391-7.

43 Herdman M, Gudex C, Lloyd A, et al. Development and preliminary testing of the new five-level version of EQ-5D (EQ-5D-5L). Qual Life Res 2011;20:1727-36.

44 Zigmond AS, Snaith RP. The hospital anxiety and depression scale. Acta Psychiatr Scand 1983;67:361-70.

45 Polonsky WH, Fisher L, Earles J, et al. Assessing psychosocial distress in diabetes: development of the diabetes distress scale. Diabetes Care 2005;28:626-31.
46 Guralnik JM, Simonsick EM, Ferrucci L, et al. A short physical performance battery assessing lower extremity function: association with self-reported disability and prediction of mortality and nursing home admission. J Gerontol 1994;49:M85-94.

47 Freiberger E, de Vreede P, Schoene D, et al. Performance-Based physical function in older community-dwelling persons: a systematic review of instruments. Age Ageing 2012;41:712-21.

48 MacDermid J, Solomon G, Fedorczyk J, et al. Clinical assessment recommendations. In: Impairment-based conditions. 3rd edition. American Society of Hand Therapists, 2015.

49 Rikli RE, Jones CJ. Senior fitness test manual. human kinetics, 2013.

50 Stenton C. The MRC breathlessness scale. Occup Med 2008:58:226-7.

51 Lee SY, Gallagher D. Assessment methods in human body composition. Curr Opin Clin Nutr Metab Care 2008;11:566-72.

52 Jeffcoate WJ, Musgrove AJ, Lincoln NB. Using image $\mathrm{J}$ to document healing in ulcers of the foot in diabetes. Int Wound $J$ 2017:14:1137-9.

53 Rowlands AV, Olds TS, Hillsdon M, et al. Assessing sedentary behavior with the GENEActiv: introducing the sedentary sphere. Med Sci Sports Exerc 2014;46:1235-47. 